\title{
Resection of esophageal diverticulum through uniportal video-assisted thoracoscopic surgery
}

\author{
Alfonso Fiorelli ${ }^{1}$, Anna Cecilia Izzo', Ettore Arrigo' ${ }^{2}$, Francesco Sgalambro ${ }^{2}$, Maria Antonietta Lepore, \\ Massimo Cajozzo ${ }^{4}$, Sergio Castorina ${ }^{5}$, Attilio Ignazio Lo Monte ${ }^{6}$, Mario Santini ${ }^{1}$, Francesco Paolo Caronia ${ }^{2}$ \\ ${ }^{1}$ Thoracic Surgery Unit, University of Campania Luigi Vanvitelli, Naples, Italy; ${ }^{2}$ Thoracic Surgery Unit, Istituto Oncologico del Mediterraneo, \\ Viagrande, Italy; ${ }^{3}$ Department of Experimental Medicine, University of Campania Luigi Vanvitelli, Naples, Italy; ${ }^{4}$ Thoracic Surgery unit, University \\ of Palermo, Palermo, Italy; ${ }^{5}$ G.B. Morgagni Foundation, Department of Bio-Medical Sciences, University of Catania, Catania, Italy; ${ }^{6}$ Dipartimento \\ DICHIRONS, University of Palermo, Palermo, Italy \\ Correspondence to: Alfonso Fiorelli, MD, PhD. Thoracic Surgery Unit, University of Campania "Luigi Vanvitelli”, Piazza Miraglia, 2, I-80138 Naples, \\ Italy. Email: alfonso.fiorelli@unicampania.it.
}

\begin{abstract}
Open surgery remains the standard strategy for management of esophageal diverticulum in symptomatic patients. However, in the last years an increasing number of minimally invasive approaches have been proposed for this issue in order to reduce the surgical trauma and favor a fast return to daily activity. Herein, we describe a novel technique as uniportal video-assisted thoracoscopic surgery (VATS) for performing resection of esophageal diverticulum. This procedure was successfully carried out in three consecutive patients with giant mid-esophageal diverticulum (mean size: $6.5 \pm 0.5 \mathrm{~cm}$ ). The mean postoperative time was $121 \pm 10$ minutes. The chest drain was removed 48 hours later in all cases and the mean length of hospital stay was $9 \pm 1$ days. No intraoperative neither postoperative complications were found in all patients but one. He had a small fistula 15 days later that was successfully treated with stent insertion. No recurrence of diverticulum was seen in all cases. Uniportal VATS is a feasible procedure that in theory could reduce the surgical trauma compared to standard open approach. However, future prospective studies should corroborate our impression before it can be recommended as acceptable therapy.
\end{abstract}

Keywords: Video-thoracoscopic; uniportal; esophageal diverticulum

Submitted Feb 05, 2018. Accepted for publication Apr 02, 2018.

doi: $10.21037 /$ atm.2018.04.12

View this article at: http://dx.doi.org/10.21037/atm.2018.04.12

\section{Introduction}

Esophageal diverticulum is a clinical entity associated with esophageal motility disorders. Surgery is indicated in patients with severe symptoms as dysphagia, esophagitis, anorexia, regurgitation and weight loss. Open surgery is the standard strategy but in the last years an increasing number of papers have reported the use of minimally invasive approaches for performing diverticulectomy and myotomy in order to reduce the surgical trauma and favor a fast return to daily activity (1-6). Herein, we describe a novel technique as the surgical treatment of midthoracic esophageal diverticulum using a uniportal video-assisted thoracoscopic surgery (VATS) approach.

\section{Study population}

In the last two years, three consecutive patients were referred to our attention for the treatment of giant midesophageal diverticulum (mean size: $6.5 \pm 0.5 \mathrm{~cm}$ ). The characteristics of study population were summarized in Table 1. All patients presented severe symptoms including dysphagia, regurgitation, gastro-esophageal reflux, food retention, weight loss and chest pain. All patients 


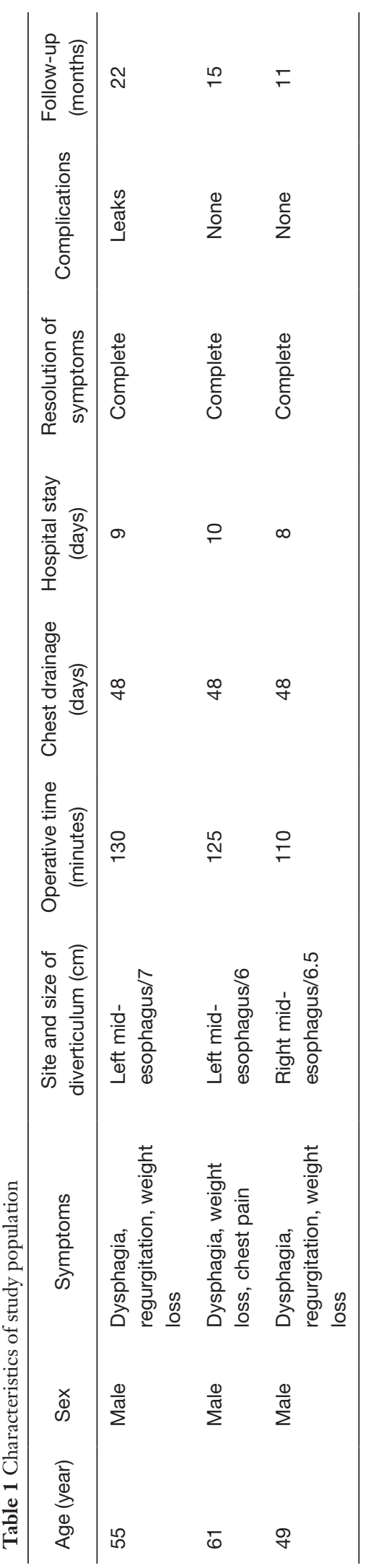

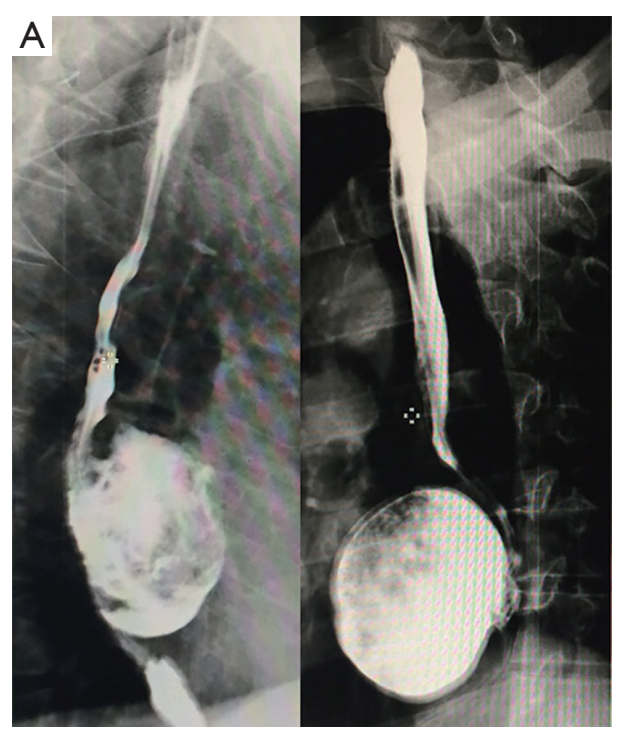

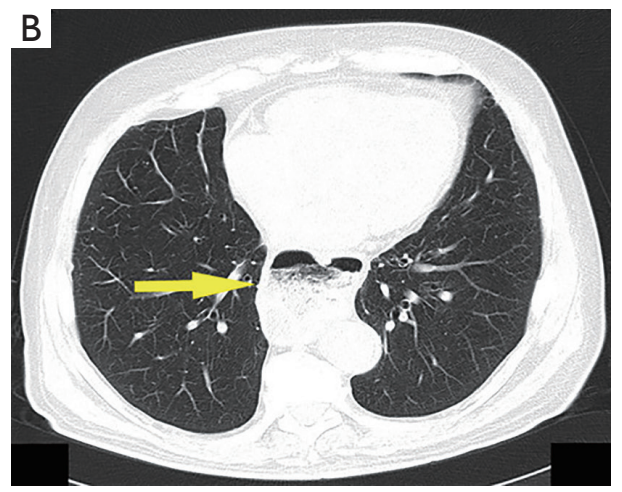

Figure 1 Gastrografin swallow (A), chest computed tomography scan (B) showed a giant mid-esophageal diverticulum. Yellow arrow shows esophageal diverticulum.

underwent standard pre-operative workup including barium swallow (Figure 1A), total body computed tomography scan (Figure 1B), manometry and 24-hour esophageal pH monitoring test. In all cases manometry showed an abnormal activity with diffuse esophageal spasm and impaired low esophageal sphincter (LES) relaxation.

All patients were aware of pros and cons of uniportal VATS approach and on the possibility of conversion to thoracotomy; they gave a specific written informed consent. Being uniportal VATS a procedure widely used in thoracic surgery, no specific approval by Institutional Review Board was required for this study.

\section{Surgical technique}

Under general anesthesia and single lung ventilation, the 

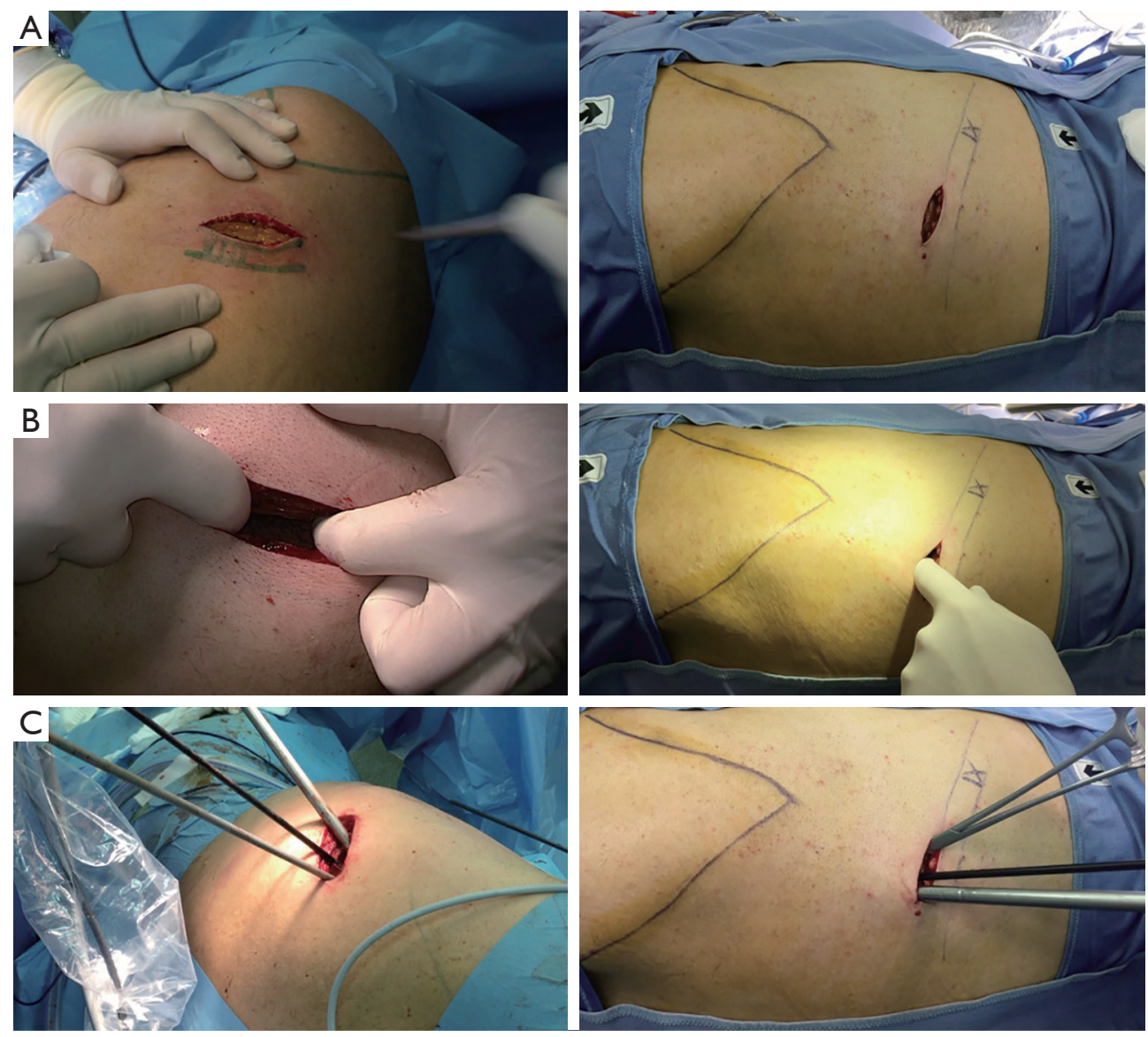

Figure 2 A 3-5-cm incision (A) was performed at level of the 7 th-8th intercostal space (B) and through that a $30^{\circ}$ camera and working instruments were inserted without rib spreading $(\mathrm{C})$.

patient was placed in the supine position. Esophagoscopy aspirated the materials in the diverticulum and a guidewire was inserted into the stomach under endoscopic view. The patient was then positioned in the left or right lateral decubitus position (according to the site of diverticulum) as for standard posterolateral thoracotomy and the left or right lung (according to the site of diverticulum) was deflated. As previously reported (7), local anesthetic (bupivacaine $10 \mathrm{~mL}$ ) was injected at level of surgical site five minutes before incision to obtain a pre-emptive analgesic effect. A 3-5-cm incision (Figure $2 A$ ) was performed at level of the right or left (according to the site of diverticulum) $7^{\text {th }}-8^{\text {th }}$ intercostal space (Figure $2 B$ ), and through that a $30^{\circ}$ camera and working instruments were inserted without rib spreading (Figure 2C).

\section{Left diverticulum}

After exploring pleural cavity, we dissected the pulmonary ligament, the mediastinal pleura over the descending aorta (Figure 3A), and the esophagus (Figure 3B) in order to expose the entire esophageal body. The diverticulum was then dissected out with careful identification of the entire neck (Figure 3C). The left crus of diaphragm was elevated and the phreno-esophageal ligament underlying cardiac fat was grasped and retracted into the thorax to expose the gastroesophageal junction. A standard extramucosal myotomy was then performed longitudinally in the anterior esophageal axis using blunt dissection and cold scissors (Figure $4 A$ ). The myotomy started $2 \mathrm{~cm}$ above the gastroesophageal junction and extended 4-8 $\mathrm{cm}$ proximally and distally involving the LES and the gastric cardia. A 50 Fr bougie was introduced across the gastroesophageal junction over the guide wire and surgeons carefully evaluated the safe passage into the esophagus. Following, the neck of diverticulum was resected at its base with a $2.5 \mathrm{~mm}$ white TA 30 stapler (Covidien, auto- 


\section{Page 4 of 7}
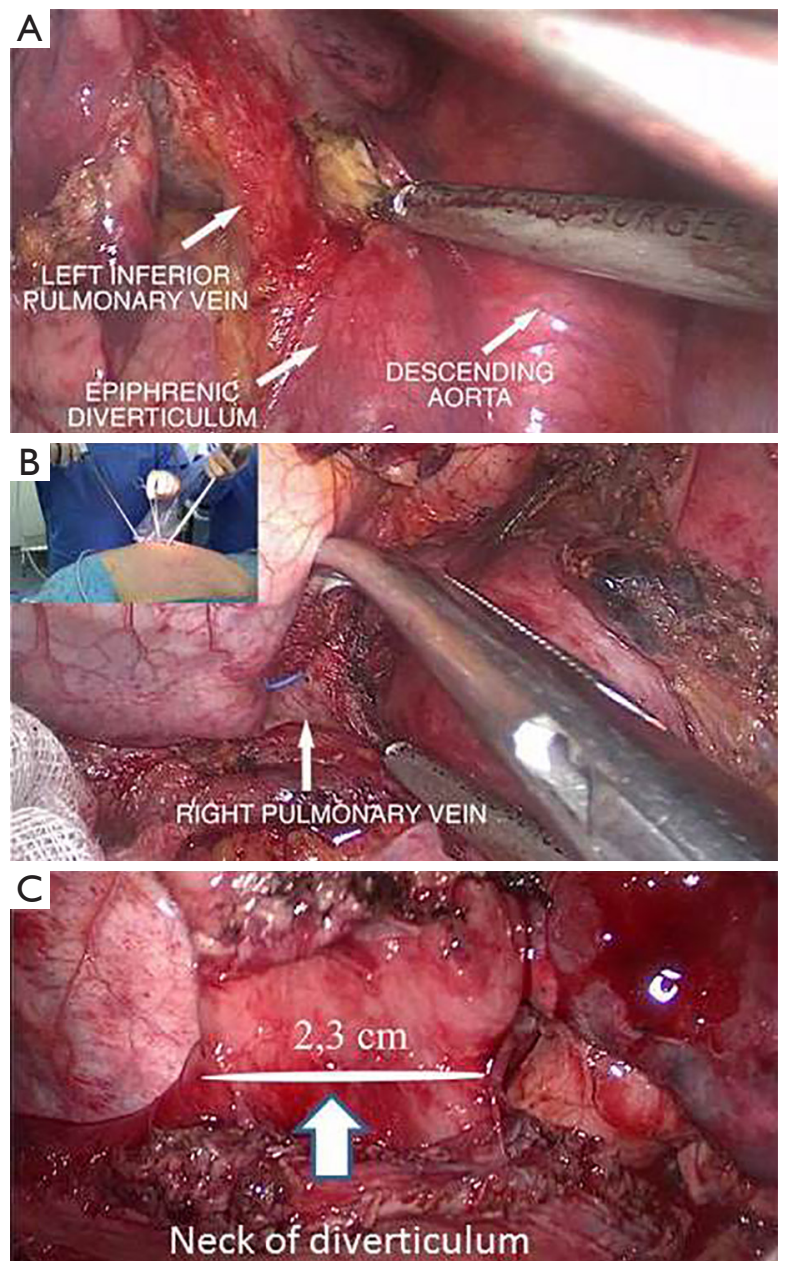

Figure 3 Dissection of mediastinal pleura over the aorta (A) and esophagus (B); complete exposition of diverticulum (C).

suture) (Figure 4B); the diverticulum was inserted into endo-bag and retrieved through the uniportal access to avoid contamination of surgical field. Finally, a BelseyMark IV fundoplication was performed in a standard manner so that the stomach, sutured to the esophagus 1 $\mathrm{cm}$ above the esophagogastric junction, encompassed $270^{\circ}$ of the esophageal circumference (Figure $5 A$ ). In the first layer, the first stitch was inserted through the esophagus and gastric fundus, $2 \mathrm{~cm}$ below the gastroesophageal junction and medially to the myotomy. The suture was tied extracorporeal and the knots descended with a standard needle holder. The second suture was placed laterally to the myotomy $2 \mathrm{~cm}$ above the gastroesophageal junction and then placed through the fundus $2 \mathrm{~cm}$ below the gastroesophageal junction. In the second row, two

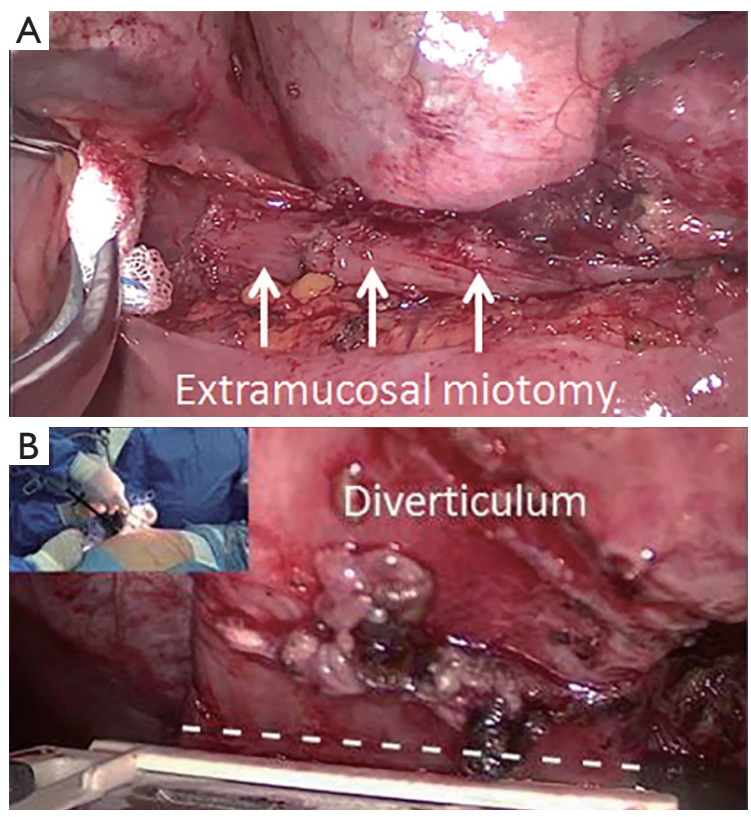

Figure 4 standard extramucosal myotomy (A) was performed and the diverticulum was then stapled at its base with an Endo GIA (B). Dashed line shows the neck of diverticulum.

sutures were placed through the esophagus medially and laterally to the myotomy, then through the stomach $4 \mathrm{~cm}$ below the gastroesophageal junction and then to the left crus intra-abdominally. The sutures were placed and tied extra corporeally anchoring the fundoplication and the gastroesophageal junction beneath the diaphragm. Finally, the suture of diverticulum was tested by insufflating air with gastroscopy under sailing. A chest drain was placed through the same uniportal incision (Figure 5B). Postoperative pain was managed with epidural analgesia in the first 24-48 hours and then with non-steroidal anti-inflammatory drugs. Oral feeding was not allowed until a contrast esophagram, performed 5-6 days postoperatively, excluded esophageal perforation. If the contrast study was normal, a clear liquid diet was initiated. Patients were discharged on a liquid diet for 2 weeks and were advanced progressively to solid food.

\section{Right diverticulum}

The procedure is similar to that of left diverticulum. The main difference was that a laparoscopic approach was needed to perform a Heller myotomy and anterior partial fundoplication as previous reported (3). 

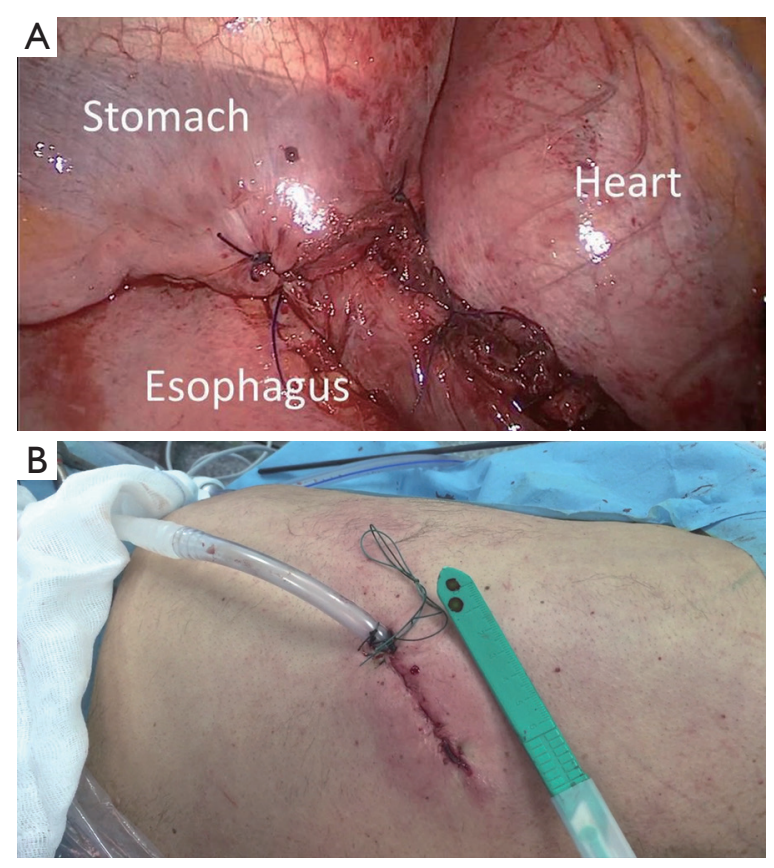

Figure 5 Standard Belsey-Mark IV fundoplication (A). Chest drainage inserted through the uniportal incision (B).

\section{Results}

In all cases the procedure was successfully performed without needing of conversion. The mean post-operative time was $121 \pm 10$ minutes. The chest drain was removed 48 hours later in all cases and the mean length of hospital stay was $9 \pm 1$ days. No intraoperative complications neither postoperative complications were found in all patients but one. He had a small fistula 15 days later that was successfully treated with stent insertion in another hospital. No recurrence of diverticulum was seen in all cases (mean follow-up: $16 \pm 5.5$ months).

\section{Discussion}

Open surgery is the treatment of choice in symptomatic patients with large esophageal diverticula. However, in the last two decades various minimally invasive techniques including laparoscopy and VATS have been developed to treat this condition (1-6). Traditional VATS is usually performed with three-four incisions but over the years there has been an evolution of VATS from multi-ports to single port. At beginning, uniportal VATS has been proposed only for minor thoracic procedures, but with the growing experience uniportal VATS has been used also to perform complex thoracic procedures (8-12) and currently there is no-doubt that every thoracic procedure could be carried out with this technique in expert hands. The less surgical trauma, less postoperative acute pain, reduction of length of hospital stay, faster return to daily activity, and better cosmetic results are all well-defined advantages of traditional VATS versus open surgery and in theory they could be also more evident if only one incision (uniportalVATS) rather than multiple-incisions (standard VATS) are made $(13,14)$.

Several authors (1-6) showed that standard VATS is a feasible and safe technique for performing diverticulectomy and myotomy, but the use of uniportal VATS for this issue remained unexplored. Recently, our group reported the first case of uniportal VATS resection of giant esophageal diverticulum (15) and this procedure was then successfully reproduced in other two patients. Conversely to standard position of uniportal VATS where the surgeon and assistant were placed anteriorly to the patient, in our technique they were positioned behind the patient and had the same surgical view of standard thoracotomy. The working instruments entered into the chest parallel to the camera but with different angulation. It allowed approaching the diverticulum from a cranio-caudal perspective similar to open surgery. Yet, the different angulation between the camera and instruments allows maximal convergence of working instruments from each side of the target lesion without interference (16). Thus, surgeon works with his eyes and hands in the same plane, similarly to the open surgery.

From a technical point of view, the following recommendations should be taken in account for the success of the procedures: (I) the first step is to resect all adhesions between the diverticulum and the mediastinal pleura in order to fully expose the neck of the diverticulum. During this maneuver great care should be taken to avoid lesion of vagus nerve and/or an excessive skeletonization of the esophagus; (II) the esophageal myotomy should be tailored to the specific motility disorder. In presence of diffuse esophageal spasm, hypertensive LES and achalasia, a long extramucosal myotomy, extending proximally for 6-8 $\mathrm{cm}$ and distally for 0.5 to $2 \mathrm{~cm}$ involving the LES and the gastric cardia, is mandatory to avoid dysphagia (17). During this maneuver caution must be taken to avoid injury to the esophageal mucosa, especially if hook cautery or harmonic scalpel are used $(18,19)$; (III) for the resection of diverticulum, the stapler should be placed at its base, parallel to the long axis of the esophagus but not perpendicular in 
order to have a regular suture without tension and reduce the risk of leak. In case of tenacious adhesions or heavy calcified lymph nodes, uniportal VATS is contraindicated and open approach should be adopted.

One of three patients presented an esophageal leak that was treated with success with a temporary stent. Suture leakage is one of the main complications of esophageal diverticulectomy that occurred with rate of $15-20 \%$ patients after minimally invasive surgery $(3,4)$.

Finally, uniportal VATS is a feasible procedure that in theory could reduce the surgical trauma and the postoperative morbidity and mortality compared to the standard open approach. However, future prospective study should corroborate our impression before it can be recommended as acceptable therapy.

\section{Acknowledgements}

None.

\section{Footnote}

Conflicts of Interest: The authors have no conflicts of interest to declare.

Informed Consent: All patients were aware of pros and cons of uniportal VATS approach and on the possibility of conversion to thoracotomy; they gave a specific written informed consent.

\section{References}

1. Saw EC, McDonald TP, Kam NT. Video-assisted thoracoscopic resection of an epiphrenic diverticulum with esophagomyotomy and partial fundoplication. Surg Laparosc Endosc 1998;8:145-8.

2. Galata CL, Bruns $\mathrm{Cj}$, Pratschke S, et al. Thoracoscopic resection of a giant midesophageal diverticulum. Ann Thorac Surg 2012;94:293-5.

3. Fernando HC, Luketich JD, Samphire J, et al. Minimally invasive operation for esophageal diverticula. Ann Thorac Surg 2005;80:2076-80.

4. Hirano Y, Takeuchi H, Oyama T, et al. Minimally invasive surgery for esophageal epiphrenic diverticulum: the results of 133 patients in 25 published series and our experience. Surg Today 2013;43:1-7.

5. Palanivelu C, Rangarajan M, Maheshkumaar GS, et al.
Minimally invasive surgery combined with peroperative endoscopy for symptomatic middle and lower esophageal diverticula: a single institute's experience. Surg Laparosc Endosc Percutan Tech 2008;18:133-8.

6. Matthews BD, Nelms CD, Lohr CE, et al. Minimally invasive management of epiphrenic esophageal diverticula. Am Surg 2003;69:465-70.

7. Fiorelli A, Vicidomini G, Laperuta P, et al. Pre-emptive local analgesia in video-assisted thoracic surgery sympathectomy. Eur J Cardiothorac Surg 2010;37:588-93.

8. Caronia FP, Fiorelli A, Santini M, et al. Extrapleural Pneumonectomy for Malignant Pleural Mesothelioma: A Novel Mini-Invasive Technique. Innovations (Phila) 2016;11:444-7.

9. Gonzalez-Rivas D, Fernandez R, Fieria E, et al. Uniportal video-assisted thoracoscopic bronchial sleeve lobectomy: first report. J Thorac Cardiovasc Surg 2013;145:1676-7.

10. Caronia FP, Fiorelli A, Santini M, et al. Uniportal bilateral video-assisted thoracoscopic extended thymectomy for myasthenia gravis: A case report. J Thorac Cardiovasc Surg 2015;150:e1-3.

11. Gonzalez-Rivas D, Fieira E, Delgado M, et al. Uniportal video-assisted thoracoscopic sleeve lobectomy and other complex resections. J Thorac Dis 2014;6:S674-81.

12. Caronia FP, Fiorelli A, Ruffini E, et al. A comparative analysis of Pancoast tumour resection performed via videoassisted thoracic surgery versus standard open approaches. Interact Cardiovasc Thorac Surg 2014;19:426-35.

13. Martin-Ucar AE, Socci $L$. Why perform uniportal videoassisted thoracic surgery?-multiple consideration. J Vis Surg 2016;2:108 .

14. Fiorelli A, Izzo AC, Frongillo EM, et al. Efficacy of wound analgesia for controlling post-thoracotomy pain: a randomized double-blind study. Eur J Cardiothorac Surg 2016;49:339-47.

15. Caronia FP, Fiorelli A, Santini M, et al. Uniportal Video-Assisted Thoracoscopic Surgery Resection of a Giant Midesophageal Diverticulum. Ann Thorac Surg 2017;103:e365-7.

16. Bertolaccini L, Rocco G, Viti A, et al. Geometrical characteristics of uniportal VATS. J Thorac Dis 2013;5 Suppl 3:S214-6.

17. Bowers SP. Esophageal motility disorders. Surg Clin North Am 2015;95:467-82.

18. Fiorelli A, Accardo M, Carelli E, et al. Harmonic technology versus neodymium-doped yttrium aluminium garnet laser and electrocautery for lung metastasectomy: 
an experimental study. Interact Cardiovasc Thorac Surg 2016;23:47-56.

19. Santini M, Fiorello A, Vicidomini G, et al. The use of LigaSure for preservation of a previous coronary artery bypass graft by using the left internal thoracic artery in a left upper lobectomy. J Thorac Cardiovasc Surg 2008;136:222-3.

Cite this article as: Fiorelli A, Izzo AC, Arrigo E, Sgalambro F, Lepore MA, Cajozzo M, Castorina S, Lo Monte AI, Santini M, Caronia FP. Resection of esophageal diverticulum through uniportal video-assisted thoracoscopic surgery. Ann Transl Med 2018;6(10):179. doi: 10.21037/atm.2018.04.12 